\title{
MULTI FRACTAL ANALYSIS OF HUMAN BRAIN MR IMAGE
}

\author{
Tarun Patil M S $\mathbf{S}^{1}$, Madhukar Bhat ${ }^{2}$ \\ ${ }^{I} U G$ Student, Department of ECE, R.V. College of Engineering, Bangalore, Karnataka, India \\ ${ }^{2} U G$ Student, Department of ECE, R.V. College of Engineering, Bangalore, Karnataka, India
}

\begin{abstract}
Brain MR Image can detect many abnormalities like tumor, cysts, bleeding, infection etc. Analysis of brain MRI using image processing techniques has been an active research in the field of medical imaging. In this work, it is shown that MR image of brain represent a multi fractal system which is described a continuous spectrum of exponents rather than a single exponent (fractal dimension). Multi fractal analysis has been performed on number of images from OASIS database are analyzed. The properties of multi fractal spectrum of a system have been exploited to prove the results. Multi fractal spectra are determined using the modified box-counting method of fractal dimension estimation.
\end{abstract}

Keywords: Brain MR Image, Multi fractal, Box-counting

\section{INTRODUCTION}

Magnetic resonance imaging and computed tomography are the two common methods used to get image of brain for diagnosis [1]. An active research has been going on in the field of medical imaging for automated detection of brain tumor using image processing techniques. With the advances in the field of technology, brain tumor imaging has become an essential component in diagnosis, treatment planning, and monitoring treatment response. Brain tumors are classified depending on the location of the tumor and the type of tissue involved.

Considerable research and application of fractal geometry has been done in image analysis and pattern recognition [2]. The brain MRI typically has a degree of randomness associated with it. This property of the image of brain is used for fractal analysis on it. Based on the fractal dimension obtained the image is analyzed for abnormalities. In this paper, brain is proved to be a multi fractal system rather than a mono fractal system.

\subsection{Background Review}

Few investigations in the past have tied to identify the fractal dimensions [3]-[6]. The limitations of the previous works were patient independent. Those limitations were due to the assumption of brain MR images as a mono fractal system. Thus we introduce multi fractal analysis which is a spectrum analysis, so the error of computation is very less. The multi fractal modelling and analysis done in our work show that the brain MRI represents a multi fractal system This result overcomes the limitations of mono fractal dimension concept in previous works.

\section{METHODOLOGY}

The key feature of the proposed method is the implementation of the algorithm in a DSP module. Fig-1 shows the schematic view of the proposed method. Code composer studio is used as an interface between PC and the TMS320C6416 DSP board. Brian MR images have been obtained from OASIS database. Histogram matching is applied to MR images to enhance the dynamic range and increase contrast. The advantage of the histogram matching is that it can have variable dynamic range of our choice. Then multi fractal modelling using modified box counting method is applied. The analysis of the fractal spectrum has been conducted extensively to prove that brain is a multi fractal system rather than a mono fractal system which is represented by a single exponent.

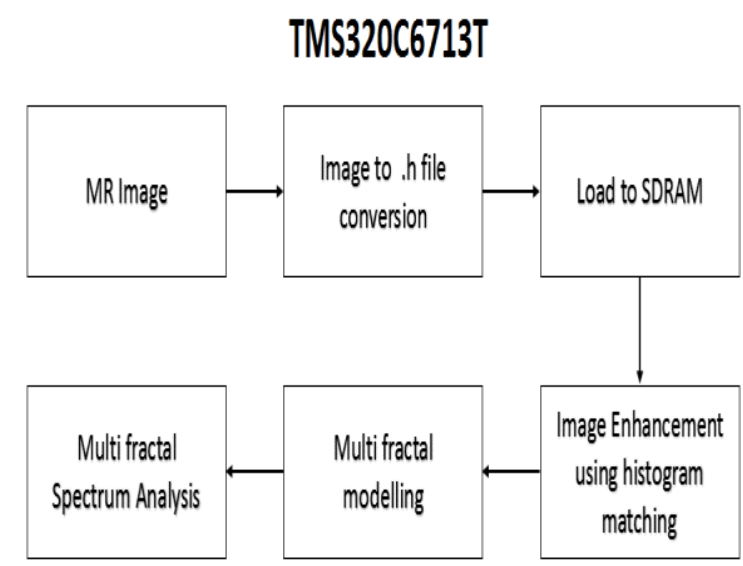

Fig -1: Schematic overview of the proposed method 


\section{FRACTAL MODELLING}

The fractal geometry concept was first proposed by Mandelbrot. A fractal is a set of iterative self similar patterns. Fractal dimension is a real number that describes the fractal property of the system. Unlike Euclidian geometry, fractal dimension can take a real value rather than just an integer. Fractal dimension value depends on the properties of the fractal system. The different methods used to calculate the fractal dimension of a system are box-counting, modified boxcounting, piecewise modified box-counting and piecewise triangular prism surface area (PTPSA). The famous fractal von Koch curve [7] shown in Fig-2 has a fractal dimension of 1.2618 .

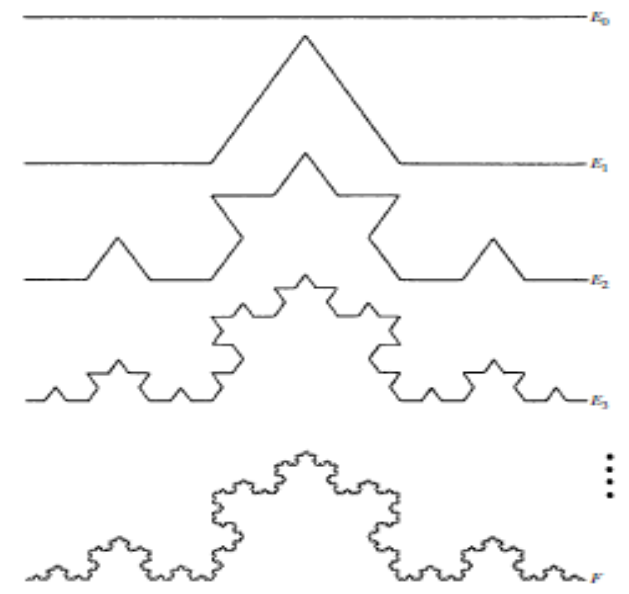

Fig -2: von Koch curve

\subsection{Fractal Dimension}

The notion of dimension is central to fractal geometry. Roughly, dimension indicates how much space a set occupies near to each of its points [8]. Fundamental to most definitions of dimension is the idea of 'measurement at scale ' $\delta$ '. For each ' $\delta$ ', we measure a set in a way that ignores irregularities of size less than $\delta$, and we see how these measurements behave as $\delta \rightarrow 0$. If $H$ is a plane curve, then our measurement, $M \delta(H)$, might be the number of steps required by a pair of dividers set at length $\delta$ to traverse $H$. A dimension of $H$ is then determined by the power law obeyed by $M \delta(H)$ as $\delta \rightarrow 0$. The dimension ' $\mathrm{s}$ ' is given by,

$$
s=\lim _{\delta \rightarrow 0}(\log M \delta(H)) /(-\log \delta)
$$

\subsection{Box Counting Dimension}

Box counting [9] is the most commonly used method to find the dimension of fractal system as it is relatively easy for mathematical calculations and empirical estimations. Let $H$ be any non-empty bounded subset of $\mathrm{R}^{\mathrm{n}}$ and let $N \delta(H)$ be the smallest number of sets of diameter at most $\delta$ which can cover $H$. The box-counting dimension of $H$ respectively are defined as,

$$
\operatorname{dimB} H=\lim _{\delta \rightarrow 0}(\log N \delta(H)) /(-\log \delta)
$$

The algorithm implemented in our work to determine the fractal dimension is,

- Cover given shape with boxes and find the pattern of change of number of boxes with the change in size of the boxes.

- If the object is 1-dimensional, we expect $\mathrm{N}$ (boxes) = $1 / \delta$.

- If the object is 2-dimensional, we expect $\mathrm{N}$ (boxes) = $(1 / \delta)^{2}$.

- For complicated shapes, the relation between $\mathrm{N}$ (boxes) and $1 / \delta$ may be a power law, $\mathrm{N}$ (boxes) $=$ $\mathrm{k}(1 / \delta)^{\mathrm{s}}$.

- $\quad$ Taking Log of both sides, we obtain $\log (\mathrm{k})$

$\log (\mathrm{N}($ boxes $))=\log (\mathrm{k})+\log \left((1 / \delta)^{\mathrm{s}}\right)=\mathrm{s} \cdot \log (1 / \delta)+$

Dimension or $\mathrm{s}=\lim (\log N($ boxes $) /(\log 1 / \delta)$

$$
\delta \rightarrow 0
$$

\subsection{Modified Box Counting Dimension}

This is the method used in our work to compute fractal dimension. There are few disadvantages of traditional box counting method. The box-counting dimension of each rational number regarded as a one-point set is clearly zero, but the countable union of these singleton sets has dimension 1 . This severely limits the usefulness of box dimension. Thus, simple modifications to suit our work has been done. For $\mathrm{H} \mathrm{a}$ subset of $\mathrm{R}^{\mathrm{n}}$ we can try to decompose $\mathrm{H}$ into a countable number of pieces $\mathrm{H} 1, \mathrm{H} 2, \ldots$ in such a way that the largest piece has as small dimension as possible.

\section{MULTI FRACTAL MODELLING}

Multi fractal patterns scale with multiple scaling rather than one scaling rule. Single parameter like fractal dimension is not enough to describe the dynamics of multi fractal system. A continuous spectrum of exponents is required to describe the system which is called the multi fractal spectrum. Multi fractal analysis was first introduced for turbulence data analysis and modelling. Now, its application in medical imaging is huge.

In multi fractal system $\mathrm{H}$, its dynamics around any point is given by,

$$
H(y+\delta)-H(y)=b^{h(y)}
$$

The exponent $\mathrm{h}(\mathrm{y})$ is called the singularity exponent. There are many methods to obtain the multi fractal spectrum like $D_{Q}$ vs $Q$ method, dimensional ordering, $f(\alpha)$ vs $\alpha$. Multi fractal 
spectrum is calculated using modified box counting in our work. Firstly, modified box counting scan is done to determine the pixels distribution. Then, this pixel distribution becomes the basis for a series of calculations. In the $f(\alpha)$ vs $\alpha$ method, the multi fractal spectrum show a parabola for the multi fractal system and random shape for non - multi fractal system. This property has been exploited to show the results.

\section{RESULTS}

In our work we have enhanced the MR Image using histogram matching method. The results are shown from Fig-3 to Fig-4.
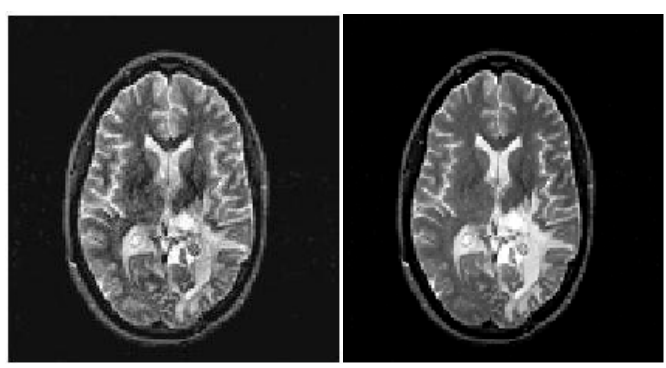

Fig -3: Original Image Fig -4: Enhanced Image
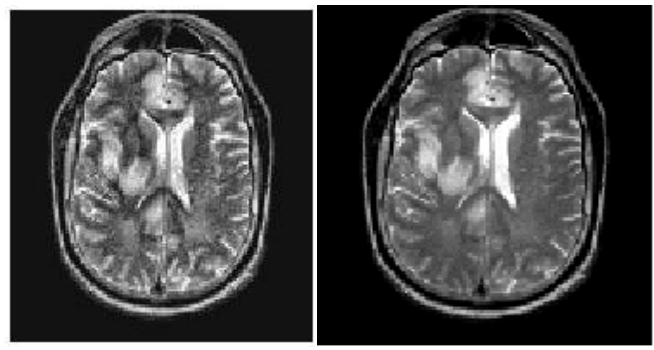

Fig -5: Original Image Fig -6: Enhanced Image

Table -1: Generalized dimension $D_{x}$ for $x=-10,10$ and standard deviation $\beta_{\mathrm{x}}$ for $\mathrm{x}=-10,10$ of 10 images from OASIS database (status: $\mathrm{N}=$ normal; $\mathrm{P}=$ pathological)

\begin{tabular}{|c|l|c|c|c|c|}
\hline $\begin{array}{c}\text { Image } \\
\text { name }\end{array}$ & status & $\mathrm{D}_{-10}$ & $\beta_{-10}$ & $\mathrm{D}_{10}$ & $\beta_{10}$ \\
\hline Img001.jpg & $\mathrm{N}$ & 1.942 & 0.015 & 1.431 & 0.003 \\
\hline Img002.jpg & $\mathrm{N}$ & 1.967 & 0.017 & 1.478 & 0.003 \\
\hline Img003.jpg & $\mathrm{N}$ & 1.939 & 0.021 & 1.440 & 0.002 \\
\hline Img007.jpg & $\mathrm{N}$ & 1.922 & 0.012 & 1.418 & 0.003 \\
\hline Img011.jpg & $\mathrm{P}$ & 1.897 & 0.018 & 1.396 & 0.001 \\
\hline Img025.jpg & $\mathrm{P}$ & 1.834 & 0.024 & 1.346 & 0.002 \\
\hline Img030.jpg & $\mathrm{N}$ & 1.926 & 0.022 & 1.432 & 0.002 \\
\hline Img040.jpg & $\mathrm{P}$ & 1.847 & 0.025 & 1.371 & 0.002 \\
\hline
\end{tabular}

\begin{tabular}{|l|l|l|l|l|l|}
\hline Img057.jpg & $\mathrm{P}$ & 1.804 & 0.019 & 1.339 & 0.003 \\
\hline Img067.jpg & $\mathrm{N}$ & 1.968 & 0.022 & 1.450 & 0.003 \\
\hline
\end{tabular}

Enhanced Images are then modelled using modified multi fractal analysis. As shown in Fig-7, alpha (q) is the range of variation in the scale of fractal measure and $\mathrm{f}(\mathrm{q})$ is the fractal dimension that we have obtained as a raw scale calculation of alpha (q). Fig-7 is the multi fractal model for the enhanced image shown in Fig-4.

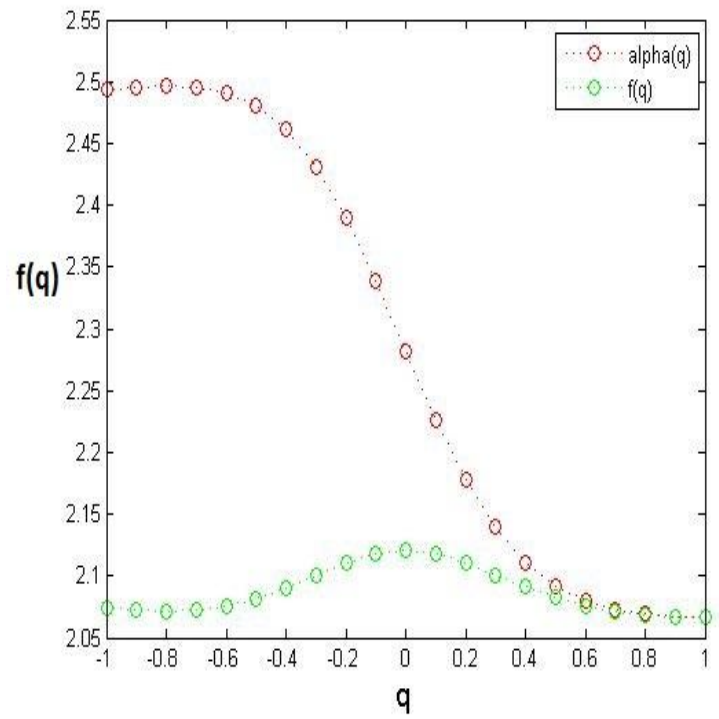

Fig -7: Variation of alpha (q) and $\mathrm{f}(\mathrm{q})$ for image in Fig-4

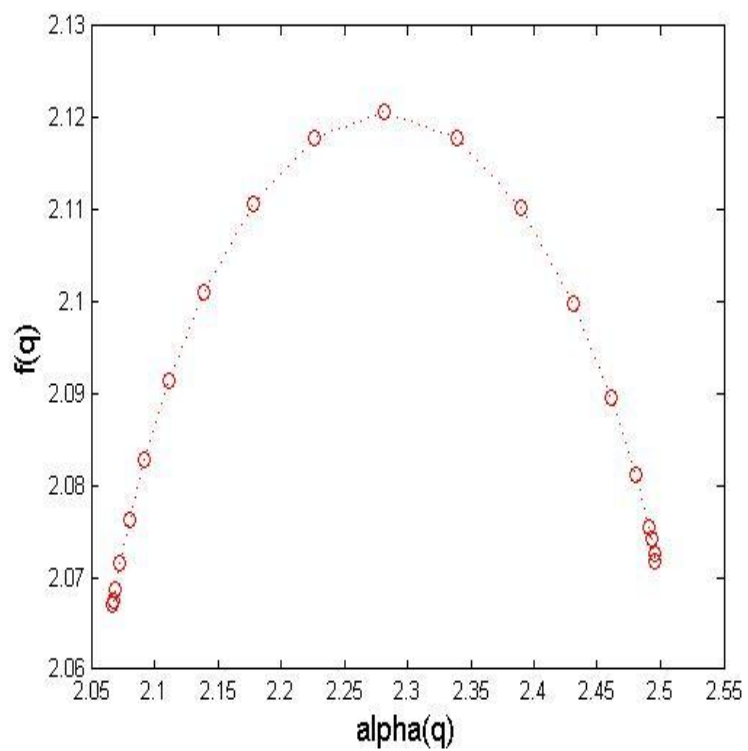

Fig -8: Multi fractal spectrum of enhanced image of Fig-4 
The above is the multi fractal spectrum of the enhanced image shown in Fig-4. It is obtained from the raw scale calculation of Fig-7. The variation of $f(q)$ with respect to the range of variation in scale of fractal dimension is plotted in Fig- 8 . The multi fractal spectrum shows a parabola for a multi fractal system. A best fit parabola to the multi fractal spectrum is plotted in Fig-9. Thus from the multi fractal spectrum shown in Fig-8-9, it can be concluded that the brain MR image shown in Fig-4 is a Multi fractal system.

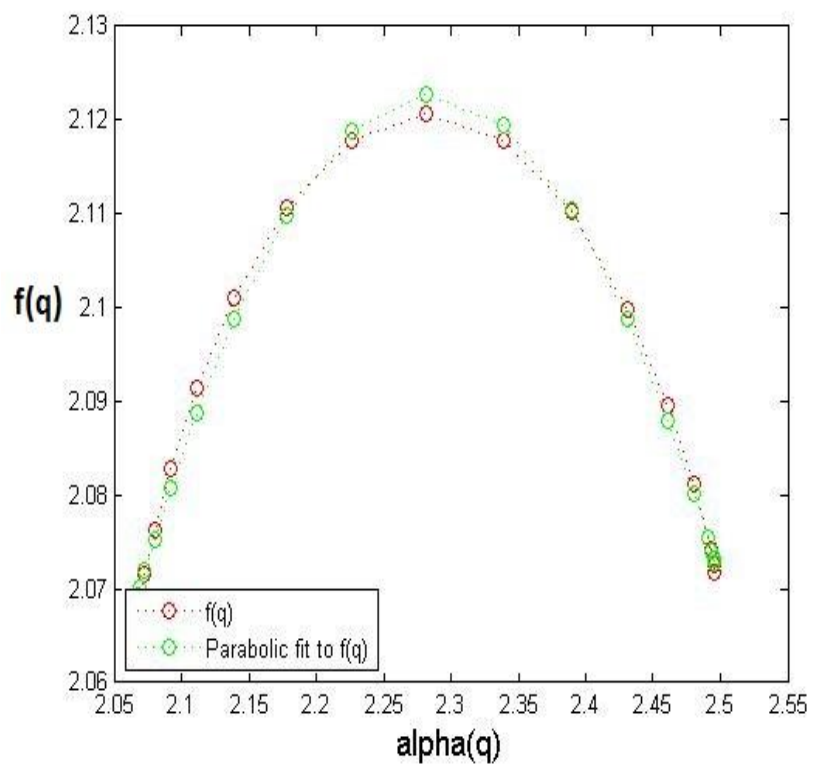

Fig -9: Best parabola fit for the multi fractal spectrum of Fig-8

\section{CONCLUSIONS}

In conclusion, we show in our work that the structure of human brain captured using MRI represent a multi fractal structure characterized by a continuous spectrum of exponents rather than a single fractal dimension. We have analyzed 40 images from the OASIS database, in which half correspond to normal brain and half correspond to abnormal brain. In all the analyzed cases, we observe a clear multi fractal behavior.

\section{ACKNOWLEDGEMENTS}

The authors would like to thank the OASIS project group for making the database publicly available.

\section{REFERENCES}

[1]. Medicine net: Magnetic Resonance Imaging (MRI scan) website: http://www.medicinenet.com/ mri_scan/article.htm [2]. Buades, Antoni, Bartomeu Coll, and Jean-Michel Morel. "A review of image denoising algorithms, with a new one" Multiscale Modeling \& Simulation 4.2 (2005): 490-530.

[3]. Tang, Min, and Hui Nan Wang. "Feature analysis of brain MRI images based on fractal dimension." Engineering in

Medicine and Biology Society, 2005 IEEE-EMBS 2005 27th Annual International Conference of the IEEE, 2006

[4]. Iftekharuddin, Khan M., et al. "Fractal-based brain tumor detection in multimodal MRI." Applied Mathematics and Computation 207.1 (2009): 23-41.

[5]. Iftekharuddin, Khan M. "Texture Models for Brain Tumor Segmentation."Quantitative Medical Imaging. Optical Society of America, 2013

[6]. Roy, Sudipta, et al. "A Review on Automated Brain Tumor Detection and Segmentation from MRI of Brain." arXiv preprint arXiv: 1312.6150 (2013).

[7]. Dorfman, J. R., and Thomas Gilbert. "Statistical properties of $\gamma$-adic processes and their connections to families of popular fractal curves (Perspectives of Nonequilibrium Statistical Physics-The Memory of Professor Shuichi Tasaki).97.3 (2011): 357-369.

[8]. Ivanovici, Mihai, and Noël Richard. "Fractal dimension of color fractal images."Image Processing, IEEE Transactions on 20.1 (2011): 227-235.

[9]. Ostwald, Michael J. "The fractal analysis of architecture: calibrating the box-counting method using scaling coefficient and grid disposition variables."Environment and Planning B: Planning and Design 40.4 (2013): 644-663.

\section{BIOGRAPHIES}
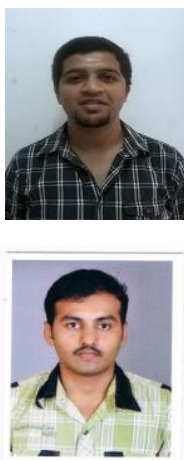

Tarun Patil M S is currently in his 8th Semester at R. V. College of Engineering, Bangalore. His research interests include medical imaging, embedded systems, Computer vision and FPGA

Madhukar Bhat is currently in his 8th Semester at R. V. College of Engineering, Bangalore. His research interests include medical imaging, Computer vision multimedia and photonic signal processing and communication systems. 